\title{
Tolerancia a la salinidad durante la germinación de semillas provenientes de poblaciones naturalizadas de agropiro alargado (Thinopyrum ponticum)
}

\author{
Omar Bazzigalupi ${ }^{1}$, Susana M. Pistorale ${ }^{2}$ y Adriana N. Andrés ${ }^{1}$ \\ ${ }^{1}$ Instituto Nacional de Tecnología Agropecuaria, Estación Experimental Agropecuaria Pergamino, \\ Casilla 31-2700 Pergamino, Buenos Aires, Argentina. \\ ${ }^{2}$ Departamento de Ciencias Básicas, Universidad Nacional de Luján, \\ Casilla 221-6700 Luján, Buenos Aires, Argentina.
}

\begin{abstract}
O. Bazzigalupi, S.M. Pistorale, and A.N. Andrés. 2008. Salinity tolerance during seed germination from naturalized populations of tall wheatgrass (Thinopyrum ponticum). Cien. Inv. Agr. 35(3):277-285. The phenotypic variability among ten adapted populations of tall wheatgrass (Thinopyrum ponticum) germinating under saline stress conditions was evaluated as part of a breeding program. The aim of the breeding program was to provide new cultivars of tall wheatgrass adapted to saline soil conditions. Saline soils represent $8 \%$ of the total agricultural soil surface in Argentina. Seeds were sown on towel paper $\left(30 \mathrm{~g} \cdot \mathrm{m}^{-2}\right)$ and germinated with alternating light and darkness ( 16 and $8 \mathrm{~h}$, respectively) and temperature (20$30^{\circ} \mathrm{C}$ ), under $0,60,135$ y $220 \mathrm{mM} \mathrm{NaCl}$ treatments, equivalent to electric conductivity of 0 , 6,12 y $18 \mathrm{dS} \cdot \mathrm{m}^{-1}$. Treatments were distributed in a randomized complete block design, with a factorial arrangement of $4 \times 11$, four salinity treatments $\times$ eleven tall wheatgrass populations; four replications were carried out, consisting of 25 seeds per experiment. The germination percentage and germination index were calculated. The results indicated large differences between populations and between treatments for both attributes. The mean germination percentage decreased $4.2,18.6$ and $61.0 \%$, as compared to the control, as electric conductivity increased to 6,12 and $18 \mathrm{dS} \cdot \mathrm{m}^{-1}$, respectively. Differences between populations were significant only in $18 \mathrm{dS} \cdot \mathrm{m}^{-1}$. The germination index had a similar performance with the increase in electric conductivity $\left(\mathrm{y}=28.827 \mathrm{x}-35.091, \mathrm{R}^{2}=0.9692\right)$. Population number 3 had the best germination and index performance. At $18 \mathrm{dS} \cdot \mathrm{m}^{-1}$, all populations had a variable number of seeds with germination capacity. Therefore, this electric conductivity could be adequate for a salt tolerance breeding and selection program.
\end{abstract}

Key words: Germination, saline stress, tall wheatgrass, Thinopyrum ponticum, variability.

\section{Introducción}

En los últimos años, se ha producido una expansión de la actividad agrícola a expensas de la superficie ocupada por la ganadería en la Pampa Húmeda y Sub-Húmeda de Argentina. Este fenómeno generó el desplazamiento de la actividad ganadera a ambientes marginales, en los que predominan pastizales $\mathrm{u}$ otras

Recibido 04 enero 2007. Aceptado 24 junio 2008.

'Dirigir correspondencia a O. Bazzigalupi: obazzigalupi@pergamino.inta.gov.ar. comunidades serales. La participación de las pasturas cultivadas en la oferta de forraje de estos sistemas ganaderos es nula, escasa o errática.

Argentina posee amplias zonas con restricciones productivas por problemas edáficos de distinta naturaleza. La salinidad afecta $320.000 \mathrm{~km}^{2} \mathrm{de}$ suelo (Maddaloni, 1986), que en la provincia de Buenos Aires representa el 24\% de su superficie agrícola. En estos ambientes, es necesario que las especies componentes de las pasturas cultivadas posean capacidad de implantación, 
elevada persistencia y adecuada oferta de nutrientes para el ganado.

Entre las diversas especies forrajeras adaptadas a los ambientes marginales templado-húmedos de Argentina se destaca Thinopyrum ponticum (Podp) Barworth et Dewey (sin. Elytrigia pontica (Podp) Holub), conocida vulgarmente como agropiro alargado. Es una gramínea perenne originaria del sur de Europa y Asia Menor, introducida en la Argentina desde EUA, en la década de los años 50. Por su rusticidad, resistencia a sequías y adaptación a suelos hidromórficos y salinos se ha difundido $\mathrm{y}$ naturalizado en los pastizales y las banquinas de la provincia de Buenos Aires.

La tolerancia a las sales es un carácter poligénico, heredable, que involucra respuestas al estrés iónico y osmótico a nivel celular (Yeo, 1998; Saleki, et al., 1993; Foolad y Jones, 1992, 1991; Cheeseman, 1988). La mayor parte de las plantas son más sensibles a la salinidad durante la germinación y emergencia que durante los estadios de crecimiento y desarrollo posteriores (Lucero, 1970; Ayers, 1950; Ayers y Haywar, 1948).

Las sales actúan en forma tóxica antes que como estímulo de la germinación de la semilla. La acción tóxica del catión o del anión puede superar al efecto producido sobre la presión osmótica (Guerrier, 1981). Además, al bajar los potenciales hídricos $(\psi)$ en el suelo, las sales bajan la tasa y la germinación total (Bradford, 1995).

Los efectos de la salinidad varían dependiendo del estadío de crecimiento y de la duración del estrés. En algunas especies, la tolerancia a la salinidad en la germinación es independiente de la tolerancia a la salinidad en la emergencia, crecimiento vegetativo, floración y fructificación. En agropiro alargado se presenta mayor sensibilidad en la etapa germinativa que en la vegetativa (Priano y Pilatti, 1989; Ferreiro y Peinemann, 1972). Junto con cebada (Hordeum vulgare), algodón (Gossypium spp.), remolacha azucarera (Beta vulgaris) y pasto miel (Paspalum dilatatum), el agropiro alargado se considera tolerante, siendo capaz de germinar y crecer en conductividades eléctricas entre 6.9 y
$8.0 \mathrm{dS} \cdot \mathrm{m}^{-1}$ (Maas y Hoffman, 1977).

Dada la característica netamente pastoril de la producción ganadera en Argentina y las limitantes edáficas de los ambientes marginales, particularmente de la Provincia de Buenos Aires, es relevante la caracterización, evaluación, y manejo de germoplasma para la obtención de nuevos cultivares. El objetivo de este trabajo fue evaluar el comportamiento germinativo de diez poblaciones de agropiro alargado, naturalizadas en la provincia de Buenos Aires, bajo estrés salino causado por cloruro de sodio $(\mathrm{NaCl})$.

\section{Materiales y métodos}

\section{Poblaciones de agropiro alargado}

Se utilizó semilla de agropiro cv. El Vizcachero (INTA), cosechada en 2002 (P11) y obtenida de diez poblaciones $(\mathrm{P})$ naturalizadas de agropiro alargado (P1 a P10) colectadas en la Cuenca del Salado, provincia de Buenos Aires. La colecta se realizó entre el 5 y el 7 de marzo de 2003. En cada sitio y población se cosecharon espigas maduras, seleccionadas en forma masal, las que se trillaron, clasificaron y almacenaron en cámara seca a $18^{\circ} \mathrm{C}$ hasta su evaluación en junio de 2003.

\section{Origen de poblaciones naturalizadas de agropiro alargado}

Se tomaron muestras de seis poblaciones de agropiro alargado, desarrolladas en suelos hidromórficos y cuatro muestras de poblaciones establecidas en suelos halo-hidromórficos (Figura 1). Estos suelos se clasifican como salino sódicos (SSSA, 1997) y se caracterizan por presentar valores máximos de $15 \%$ de sodio intercambiable, $\mathrm{pH} 9$ a pH 10 y 10 a $12 \mathrm{dS} \cdot \mathrm{m}^{-1} \mathrm{de}$ conductividad eléctrica (INTA-CIRN, 1989).

\section{Tratamientos}

Las semillas, superficialmente desinfectada con hipoclorito de sodio al $5 \%$ por $3 \mathrm{~min}$, se sembraron en bandejas de polietileno, sobre una doble capa de toalla de papel $\left(30 \mathrm{~g} \cdot \mathrm{m}^{-2}\right)$, humedecido con soluciones 60, 135 y 220 $\mathrm{mM}$ de $\mathrm{NaCl}$, equivalentes a conductividades 


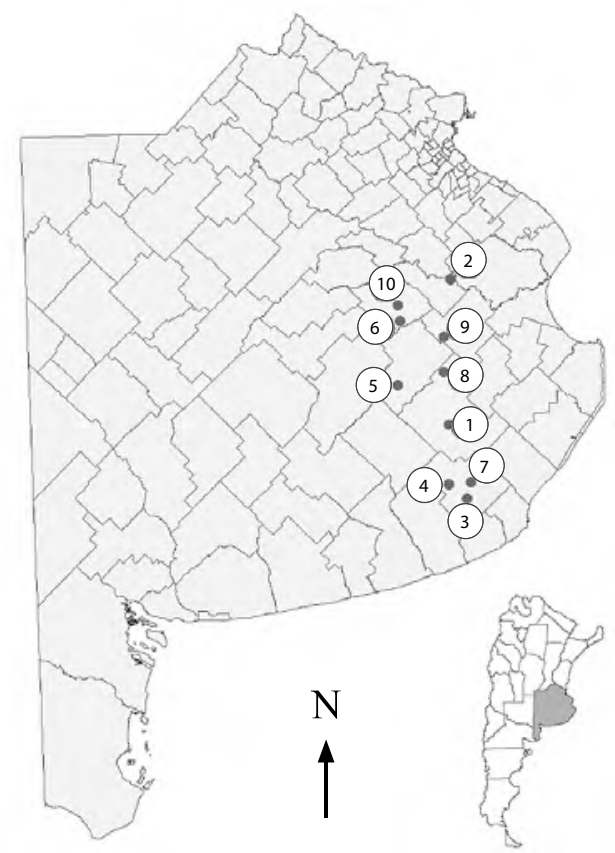

Figura 1. Distribución geográfica de poblaciones naturalizadas de Thinopyrum ponticum en la Cuenca del Salado (36-38 $\left.\mathrm{S}, 62-58^{\circ} \mathrm{O}\right)$ de la Provincia de Buenos Aires, Argentina.

Figure 1. Geographic distribution of naturalized populations of tall wheatgrass (Thinopyrum ponticum) in the Salado Basin $\left(36^{\circ}-38^{\circ} \mathrm{S}, 62^{\circ}-58^{\circ} \mathrm{W}\right)$ in the Buenos Aires Province, Argentina.

eléctricas de 6,12 y $18 \mathrm{dS} \cdot \mathrm{m}^{-1}$. Como testigo se utilizó agua destilada con una conductividad eléctrica igual a $0 \mathrm{dS} \cdot \mathrm{m}^{-1}$ (Cuadro 1). Cada bandeja se introdujo en bolsa de polietileno transparente (20 $\mathrm{m} \mu$ de espesor), para reducir la evaporación y mantener la concentración de la solución salina. Se incubaron en cámara de germinación con equipamiento de frío y calor (Pasti Ingeniería, Pergamino, Argentina). Los estantes estuvieron separados $45 \mathrm{~cm}$, con temperatura 20 a $30^{\circ} \mathrm{C} \pm 1,1$, en ciclos diarios de $16 \mathrm{~h}$ de oscuridad y $8 \mathrm{~h}$ de luz $(250 \mu \mathrm{mol}$ photón $\cdot \mathrm{m}^{-2} \cdot \mathrm{seg}^{-1}$, respectivamente)(ISTA, 1999).

\section{Evaluación}

Se determinó la proporción de semilla germinada, sensu stricto, a los 5, 11, 19 y 25 d después de la siembra. Para cada población de agropiro alargado, los resultados obtenidos se expresaron porcentualmente en relación con el testigo sin tratar (Maas, 1986; Maas y Hoffman, 1977).

El efecto de los tratamientos también se evaluó mediante un índice de germinación (IG) variable entre 0 y 5 para expresar la rapidez de la germinación, siendo 0 baja rapidez y 5 de alta rapidez de germinación. El IG se calculó de acuerdo con la siguiente ecuación:

$$
\mathrm{IG}=\sum \mathrm{Gi} / \mathrm{Di}
$$

donde Gi fue la diferencia en número de semillas germinadas entre el recuento $i$, y el recuento precedente; Di fue el día de cada recuento expresado en d después de la siembra $(5,11,19$ y 25 d).

\section{Diseño y análisis estadísticos}

El diseño experimental fue en bloques completos aleatorizados, con arreglo factorial $4 \times 11$, cuatro tratamientos de salinidad $\mathrm{x}$ once poblaciones de agropiro alargado, con cuatro repeticiones de 25 semillas en cada unidad experimental. Los resultados se analizaron para varianza y los promedios se separaron de acuerdo con la prueba de Tukey, empleando SAS 2008 (SAS Institute Inc., Cary, NC, EUA). Previo a los análisis estadísticos, los valores porcentuales se transformaron en valores angulares $(\operatorname{arcoseno}(\sqrt{ } \mathrm{x})$.

Cuadro 1. Concentración, conductividad eléctrica, potencial osmótico y $\mathrm{pH}$ a $25^{\circ} \mathrm{C}$ de las soluciones de $\mathrm{NaCl}$ empleadas en los ensayos de germinación.

Table 1. Concentration, electric conductivity, osmotic potential, and $\mathrm{pH}$ of the $\mathrm{NaCl}$ solutions used in the germination tests at $25^{\circ} \mathrm{C}$.

\begin{tabular}{lccc}
\hline $\begin{array}{l}\text { Concentración } \\
\text { de } \mathrm{NaCl}\end{array}$ & $\begin{array}{c}\text { Conductividad } \\
\text { eléctrica } \\
\mathrm{mM}\end{array}$ & $\begin{array}{c}\text { Potencial } \\
\text { osmótico } \\
\mathrm{dS} \cdot \mathrm{m}^{-1}\end{array}$ & $\mathrm{pH}$ \\
\hline 0 & 0 & 0,00 & 6,4 \\
60 & 6 & $-0,27$ & 6,5 \\
135 & 12 & $-0,62$ & 6,5 \\
220 & 18 & $-1,01$ & 5,9 \\
\hline
\end{tabular}




\section{Resultados}

\section{Germinación}

Por falta de homogeneidad de varianza se excluyeron del análisis estadístico el tratamiento testigo $\left(0 \mathrm{dS} \cdot \mathrm{m}^{-1}\right)$ y la población $\mathrm{P} 4$. No se detectó dormición de semillas.

En relación con el testigo, la disminución promedio de la germinación obtenida fue $4.2,18.6$ y $61.0 \%$ en semillas tratada con las soluciones de de $\mathrm{NaCl}$ equivalentes a 6, 12 y 18 $\mathrm{dS} \cdot \mathrm{m}^{-1}$, respectivamente. La interacción entre salinidad y poblaciones de agropiro alargado fue significativa $(\mathrm{p}=0,0337)$. Por lo tanto, el comportamiento de las poblaciones de agropiro alargado se analizó independientemente en cada una de las tres soluciones salinas. La disminución del número de semillas germinadas por incremento en la conductividad eléctrica de las soluciones varió entre las poblaciones de agropiro alargado (Cuadro 2).

En soluciones con 6 y $12 \mathrm{dS} \cdot \mathrm{m}^{-1}$ no hubo diferencias significativas en germinación entre las poblaciones de agropiro alargado. No obstante, se obtuvieron diferencias significativas en germinación en semillas expuestas a 18 $\mathrm{dS} \cdot \mathrm{m}^{-1}$ (Cuadro 2).

Se destacó la población P3, con menor reducción de germinación respecto al testigo $\mathrm{y}$ fue consistentemente diferente de las poblaciones P1, P8, P2, P11 y P6, pero sin diferencias significativas $(p=0,05)$ respecto de las poblaciones P10, P9, P5 y P7 (Cuadro 2).

Las poblaciones P5, P9 y P10 fueron colectadas en suelos hidro-halomórficos, mientras que la población P3 se colectó en un suelo hidromórfico, y fue la población que presentó la mayor germinación (64\% relativo al testigo) en $18 \mathrm{dS} \cdot \mathrm{m}^{-1}$ de conductividad eléctrica.

Cuadro 2. Efecto de la salinidad, expresado a través de la conductividad eléctrica, sobre la germinación relativa al testigo $\left(0 \mathrm{dS} \cdot \mathrm{m}^{-1}\right)$ de las poblaciones $(\mathrm{P})$ de agropiro alargado (Thinopyrum ponticum) determinado $25 \mathrm{~d}$ después de la siembra.

Table 2. Effect of salinity, expressed as electrical conductivity, on seed germination relative to the check $\left(0 \mathrm{dS} \cdot \mathrm{m}^{-1}\right)$ in tall wheatgrass (Thinopyrum ponticum) populations $(P)$, determined $25 d$ after sowing.

\begin{tabular}{|c|c|c|c|c|}
\hline \multirow[t]{2}{*}{$\begin{array}{l}\text { Población de } \\
\text { agropiro alargado }^{1}\end{array}$} & \multicolumn{4}{|c|}{$\begin{array}{l}\text { Germinación }(\%) \text { en tratamientos con } \mathrm{NaCl} \text { equivalentes a } \\
\text { conductividades eléctricas }\left(\mathrm{dS} \cdot \mathrm{m}^{-1}\right) \text { de: }\end{array}$} \\
\hline & 0 & 6 & 12 & 18 \\
\hline P3 & 100 & $97,0 \mathrm{a}^{2}$ & $93,0 \mathrm{a}^{2}$ & $64,0 \mathrm{a}^{2}$ \\
\hline P10 & 100 & $99,0 \mathrm{a}$ & $91,0 \mathrm{a}$ & $57,0 \mathrm{ab}$ \\
\hline P9 & 100 & $97,0 \mathrm{a}$ & $82,0 \mathrm{a}$ & $48,0 \mathrm{abc}$ \\
\hline P5 & 100 & $97,0 \mathrm{a}$ & 87,0 a & $47,0 \mathrm{abc}$ \\
\hline P7 & 100 & $93,0 \mathrm{a}$ & $82,0 \mathrm{a}$ & $45,0 \mathrm{abc}$ \\
\hline P1 & 100 & $97,0 \mathrm{a}$ & $87,0 \mathrm{a}$ & $31,0 \mathrm{bc}$ \\
\hline P8 & 100 & 97,0 a & 86,0 a & $28,0 \mathrm{c}$ \\
\hline $\mathrm{P} 2$ & 100 & $90,0 \mathrm{a}$ & $72,0 \mathrm{a}$ & $27,0 \mathrm{c}$ \\
\hline P11 & 100 & $99,0 \mathrm{a}$ & $69,0 \mathrm{a}$ & $27,0 \mathrm{c}$ \\
\hline P6 & 100 & $98,0 \mathrm{a}$ & $71,0 \mathrm{a}$ & $21,0 \mathrm{c}$ \\
\hline Media & 100 & 95,8 & 81,4 & 39,0 \\
\hline \multicolumn{5}{|l|}{ Análisis de varianza: } \\
\hline Fuente de variación & & & $\mathrm{F}$ & $\mathrm{p}$ \\
\hline Bloques & & & 2,00 & 0,1201 \\
\hline Concentración salina (CS) & & & 179,64 & $<0,0001$ \\
\hline Poblaciones $(\mathrm{P})$ & & & 5,11 & $<0,0001$ \\
\hline $\mathrm{CS} \times \mathrm{P}$ & & & 1,83 & 0,0337 \\
\hline Residual & & 59. & & \\
\hline
\end{tabular}

${ }^{1}$ Se excluyó la población P4 por falta de homogeneidad de varianza.

${ }^{2}$ Medias seguidas iguales letras, dentro de cada columna, no son significativamente diferentes según la comparación múltiple de Tukey ( $\left.\mathrm{p}=0,05\right)$.

${ }^{1}$ Population P4 was excluded because of the absence of variance homogeneity.

${ }^{2}$ Meansfollowed by the same letter withineach columns are not significantlydifferent according to the multicomparison test of Tukey $(p=0.05)$. 


\section{Indice de germinación}

El tratamiento testigo $\left(0 \mathrm{dS} \cdot \mathrm{m}^{-1}\right)$ se excluyó del análisis estadístico por falta de homogeneidad de varianza.

A diferencia de la germinación, para el IG la interacción entre poblaciones de apropiro alargado y tratamientos de salinidad no fue significativa $(\mathrm{p}=0,1353)$. En todas las poblaciones de agropiro alargado, el IG se redujo de manera significativa al aumentar la concentración salina, siendo muy drástico su efecto en el tratamiento equivalente a 18 $\mathrm{dS} \cdot \mathrm{m}^{-1}$ de conductividad eléctrica (Cuadro 3 ).
Las poblaciones P3 y P10 tuvieron la mayor rapidez de germinación, superando en forma consistente a las poblaciones P2, P4, P6, P7 y $\mathrm{P} 11$. En el grupo intermedio se ubicaron las poblaciones P5, P1, P9 y P8 (Cuadro 3).

La reducción de la rapidez de germinación de las poblaciones respecto al testigo mostró un comportamiento lineal con el incremento en la concentración de las soluciones $(\mathrm{y}=28,827 \mathrm{x}$ 35,$\left.091 ; \mathrm{R}^{2}=0,9692\right)$. Las poblaciones P3 y P10 tuvieron la más baja reducción del IG en contraste con las poblaciones P6 y P7 que presentaron una más alta reducción del IG (Figura 2).

Cuadro 3. Indice de germinación de la siembra de agropiro alargado (Thinopyrum ponticum) determinado a los $25 \mathrm{~d}$.

Table 3. Germination index of tall wheatgrass (Thinopyrum ponticum) determined $25 \mathrm{~d}$ after sowing.

Promedio de las poblaciones de agropiro alargado:

$\begin{array}{cc}\text { Tratamientos salinos } & \text { Indice de germinación } \\ \text { conductividad eléctrica } & \text { escala de } 0 \text { a } 5^{1}\end{array}$
$\mathrm{dS} \cdot \mathrm{m}^{-1}$

\begin{tabular}{lcl}
\hline & 6 & $3,948 \mathrm{a}^{2}$ \\
& 12 & $2,414 \mathrm{~b}$ \\
& 18 & $0,759 \mathrm{c}$ \\
\hline
\end{tabular}

Promedio de los tratamientos salinos:

\begin{tabular}{lcc} 
& \multicolumn{2}{c}{$\begin{array}{c}\text { Indice de germinación } \\
\text { escala de 0 a 5 }\end{array}$} \\
\cline { 2 - 3 } Poblaciones de agropiro alargado & $\begin{array}{c}\text { Testigo } \\
0 \mathrm{dS} \cdot \mathrm{m}^{-1}\end{array}$ & $\begin{array}{c}\text { Promedio de } \\
\text { los tratamientos }\end{array}$ \\
\hline P3 & 5,00 & $3,196 \mathrm{a}^{2}$ \\
P10 & 4,96 & $2,971 \mathrm{a}$ \\
P5 & 5,00 & $2,762 \mathrm{ab}$ \\
P1 & 4,90 & $2,687 \mathrm{ab}$ \\
P9 & 4,84 & $2,668 \mathrm{ab}$ \\
P8 & 4,79 & $2,528 \mathrm{abc}$ \\
P2 & 4,82 & $2,148 \mathrm{bcd}$ \\
P4 & 4,33 & $1,883 \mathrm{~cd}$ \\
P6 & 4,23 & $1,859 \mathrm{~cd}$ \\
P7 & 4,68 & $1,771 \mathrm{~d}$ \\
P11 & 3,41 & $1,619 \mathrm{~d}$ \\
\hline
\end{tabular}

Análisis de varianza:

\begin{tabular}{lrrrr} 
Fuente de variación & g.l. & CM & F & P \\
\hline Bloques & 3 & 262,40 & 3,45 & 0,0196 \\
Concentración salina (CS) & 2 & 24657,70 & 324,30 & $<0,001$ \\
Poblaciones (P) & 10 & 382,90 & 5,04 & $<0,001$ \\
P x CS & 20 & 107,40 & 1,41 & 0,1353 \\
Residual & 96 & 76,03 & & \\
\hline
\end{tabular}

${ }^{1}$ Indice de germinación, 0 = lento y 5 = rápido.

${ }^{2}$ Promedios seguidos de la misma letra no son estadísticamente diferentes según la prueba de Tukey $(\mathrm{p}<0,05)$.

${ }^{1}$ Germination index, $0=$ show and 5 = fast.

${ }^{2}$ Means followed by the same letters are not significantly different according to Tukey's test $(p<0.05)$. 

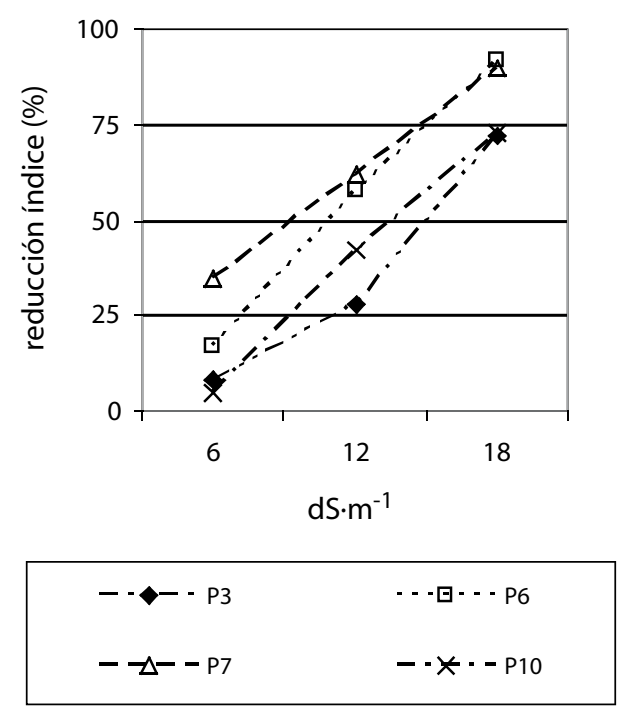

Figura 2. Porcentaje de reducción del índice de germinación $(0=$ lento y $5=$ rápido $)$ respecto al testigo (0 dS.m-1), correspondiente a las poblaciones P3, P6, P7 y P10 de Thinopyrum ponticum. Rango del índice entre 0 (germinación lenta) y 5 (germinación rápida).

Figure 2. Relation between the electrical conductivity and germination index $(0=$ slow and $5=$ fast $)$ obtained for the seed of tall wheatgrass (Thinopyrum ponticum) populations $\mathrm{P} 3, \mathrm{P} 6, \mathrm{P7}$, and $\mathrm{P} 10$.

\section{Discusión}

El estrés salino es una condición adversa a las plantas muy prevalente en Argentina. El impacto negativo provocado por la salinidad aumenta a medida que la agricultura se desplaza hacia zonas marginales o como resultado del inadecuado manejo de los agroecosistemas. La mejora genética de las especies de pastos adaptados a suelos salinos es una estrategia de control válida.

De acuerdo con los resultados obtenidos, la tasa de germinación se relacionó con el potencial hídrico $(\psi)$ del sustrato de incubación y con el potencial de base de la semilla $(\psi b)$. Estos influyen en la imbibición de agua, en la duración del período de reposo previo a la emergencia de la radícula (germinación sensu stricto) y determinan la germinación total de la población de semillas. La disminución del $\psi$ en la solución explica la demora progresiva en la germinación, el incremento en la variación de los tiempos de germinación entre semillas y la variación en el porcentaje final (Bradford, 1995).

La metodología empleada en este trabajo no permitió despejar el efecto de los iones del efecto de los $\psi$ de las soluciones. Sin embargo, varios autores refieren sobre la eficacia de soluciones de $\mathrm{NaCl}$ para evaluar la adaptación de las especies a la salinidad (Guerrier, 1981).

Cuando las semillas son colectadas de poblaciones naturales y usadas en estudios de ecología de la germinación, las diferencias en sus respuestas pueden incluir componentes genéticos y ambientales (Quinn y Colosi, 1977). En especies forrajeras se ha demostrado la existencia de variabilidad genética heredable respecto a la tolerancia a salinidad, en especial cuando se evalúan poblaciones provenientes de lugares con limitaciones edáficas (Rogers et al., 1998; Shannon y Noble, 1995; Rumbaugh y Pendery, 1990; Horst y Dunning, 1989; Marcar, 1987; et al., 1986c, 1987;Allen et al., 1985; Ando et al., 1985).

En varios ensayos con especies gramíneas, Ashraf (1994) evaluó la variabilidad de la tolerancia a la salinidad en poblaciones nativas obtenidas en sitios contrastantes en salinidad edáfica. Las poblaciones provenientes de lugares salinos fueron las más tolerantes a la salinidad; sin embargo, las de lugares no salinos presentaron variabilidad para dicho carácter. Es posible que la tolerancia este subyacente en dichas poblaciones debido a una falta de selección natural. Esto podría explicar el mejor comportamiento germinativo de la población P3.

Para el conjunto de las poblaciones, las soluciones de -0.27 y -0.6 MPa (6 y $\left.12 \mathrm{dS} \cdot \mathrm{m}^{-1}\right)$, no permitieron discriminar por aptitud para germinar. Sólo la solución de -1.0 MPa (18 $\mathrm{dS} \cdot \mathrm{m}^{-1}$ ) permitió discriminar entre poblaciones. Estos resultados son comparables con resultados previamente obtenidos en agropiro alargado, donde se encontró evidencias de una brusca disminución del crecimiento de las plántulas, con disminución del largo de las raíces asociadas al efecto de una conductividad eléctrica de $18 \mathrm{dS} \cdot \mathrm{m}^{-1}$ (Roundy, 1983). 
Todas las poblaciones de agropiro alargado presentaron una proporción variable de semillas con capacidad de germinar en el potencial de -1.0 MPa, con 25 d de incubación. Significaría que ese potencial es adecuado para trabajar con alta presión de selección en agropiro alargado para el carácter evaluado.

Aún en agua (0,0 MPa) no todas las semillas germinan simultáneamente, existiendo diferencias en los $\psi \mathrm{b}$ entre semillas de la misma población. La distribución en el tiempo es un indicador de la uniformidad del lote de semillas. Cuando disminuye el potencial de la solución aumenta en forma progresiva el tiempo para alcanzar un determinado porcentaje de germinación. Las semillas más lentas son las que presentan valores de $\psi \mathrm{b}$ más altos (Bradford, 1995).

El índice de germinación permitió una mayor diferenciación entre las poblaciones de agropiro alargado. Tanto para el índice como para la germinación se destacan las poblaciones P3 y P10, probablemente con mayor cantidad de semillas con menores $\psi \mathrm{b}$ o mecanismos metabólicos para neutralizar eventuales efectos tóxicos del ión sodio.

En coincidencia con Miller y Chapman (1978) los resultados de este trabajo muestran que el aumento de las conductividades influye más en el índice de germinación que en la germinación total de la semilla.

Según Blum (1988), Norlyn y Epstein (1984) y Greenway (1965) la evaluación de materiales en germinación y estado de plántula son prácticos y efectivos para identificar material tolerante a salinidad. En este sentido, Ashraf et al. (1986a,b) y Shannon (1979), opinan que es importante evaluar la tolerancia en todas las etapas de desarrollo.

El interés por mejorar la tolerancia de los cultivos a la salinidad ha crecido en los últimos años, ya sea mediante métodos de mejora y selección tradicionales como mediante la producción de organismos genéticamente modificados. Diferentes alternativas se pueden implementar para mejorar la aptitud de los suelos afectados por condiciones de salinidad.
Muchas de ellas son extremadamente costosas y no solucionan en forma permanente el problema. En contraste, la aproximación biológica ha ganado considerable reconocimiento debido a la evolución de la tolerancia a la salinidad en ecotipos de diferentes especies vegetales (Pearen et al., 1997). Por otra parte, existe un importante cúmulo de información acerca de los efectos de la salinidad sobre el desempeño de los cultivos, que brindan una acabada disección de sus componentes fisiológicos, bioquímicos y moleculares (Ma et al., 2006; Munns et al., 2006; Munns, 2005, 2002; Flowers, 2004; Hasegawa et al., 2000).

De acuerdo con los resultados obtenidos, en ambiente salino las poblaciones naturalizadas de agropiro alargado poseen variabilidad en su capacidad de germinar. Por otra parte, existiría algún grado de asociación entre el lugar de recolección de las poblaciones y su capacidad para germinar en ambiente salino. En los programas de selección por germinación en estrés salino por $\mathrm{NaCl}$ se deberían utilizar potenciales inferiores a $-0,62 \mathrm{MPa}\left(12 \mathrm{dS} \cdot \mathrm{m}^{-1}\right)$. Con potenciales superiores a $-0,6 \mathrm{MPa}(12$ $\mathrm{dS} \cdot \mathrm{m}^{-1}$ ) no se alcanzaría una eficaz presión de selección; mientras que, soluciones de $-1 \mathrm{MPa}$ $\left(18 \mathrm{dS} \cdot \mathrm{m}^{-1}\right)$ serían adecuadas para lograrla, debido a que todas las poblaciones de agropiro alargado presentaron una cantidad variable de semillas con capacidad para germinar en ese potencial hídrico.

\section{Resumen}

La variabilidad fenotípica de diez poblaciones naturalizadas de agropiroalargado (Thinopyrum ponticum), fue evaluada en un programa de selección cuya finalidad es proveer cultivares adaptadas a suelos salinos, que en Argentina representan el $8 \%$ de la superficie agropecuaria total. Las poblaciones se colectaron en la pampa deprimida, Buenos Aires, Argentina El objetivo fue evaluar su germinación en estrés salino por soluciones de $\mathrm{NaCl} 0,60,135$ y 220 $\mathrm{mM}$ equivalentes a conducitividad eléctrica de $0,6,12$ y $18 \mathrm{dS} \cdot \mathrm{m}^{-1}$. Las semillas fueron sembradas sobre toallas de papel e incubadas en cámara de germinación con alternancia diaria de temperatura $\left(20-30^{\circ} \mathrm{C}\right)$ y luz $(16-8$ h). Se empleó un diseño en bloques completos 
aleatorizados con arreglo factorial, con cuatro repeticiones de 25 semillas. A los 5, 11, 19 y 25 días se realizaron recuentos para calcular porcentaje y velocidad de germinación. El número de semillas germinadas disminuyó con el incremento de la conductividad, en todas las poblaciones, con diferencia consistentes entre estas sólo en $18 \mathrm{dS} \cdot \mathrm{m}^{-1}$. Respecto al testigo, la germinación se redujo 4,2; 18,6 y $61,0 \%$ en las soluciones de 6,12 y $18 \mathrm{dS} \cdot \mathrm{m}^{-1}$ respectivamente $\mathrm{y}$ el índice, en forma lineal con el incremento en la conductividad de las soluciones $(\mathrm{y}=28,827 \mathrm{x}$ 35,$\left.091 ; \mathrm{R}^{2}=0,9692\right)$. La población P3 expresó el mejor comportamiento germinativo. En soluciones con $18 \mathrm{dS} \cdot \mathrm{m}^{-1}$ todas las poblaciones presentan semillas con capacidad de germinar. Así, en programas de mejoramiento genético, esta conductividad sería adecuada para seleccionar materiales y para informar sobre el rango de respuesta adaptativa al ambiente.

Palabras clave: Agropiro alargado, estrés osmótico, germinación, salinidad, Thinopyrum ponticum, variabilidad.

\section{Agradecimientos}

Los autores agradecen a Sergio Fontana, Ana Font, Catalina Améndola, Adriana Ferreira e Iris Ballestrase, de la Estación Experimental INTA Pergamino, Buenos Aires, Argentina, por su colaboración en diferentes etapas del trabajo. Así como al Departamento de Ciencias Básicas de la Universidad Nacional de Luján (Buenos Aires, Argentina) por el financiamiento recibido para la realización de parte de este trabajo.

\section{Literatura citada}

Allen, S.G., A.K. Dobrenz, M.H. Schonhorst y J.E. Stoner. 1985. Heritability of $\mathrm{NaCl}$ tolerance in germinating alfalfa seeds. Agronomy Journal 77:99-101.

Ando,T., Y. Masoka y K. Matsumoto. 1985. Interspecific differences in sodiumaccumulation and requirement among forage crops. Soil Science and Plant Nutrition 31:601-610.

Ashraf, M. 1994. Breeding for salinity tolerance in plant. Critical Reviews in Plant Sciences 13:1742.

Ashraf, M., T. Mcneilly y A.D. Bradshaw. 1986a. Heritability of sodium chloride tolerance in seven grass species. Euphytica 35:935-940.

Ashraf, M., T. Mcneilly y A.D. Bradshaw. 1986b.
The response of selected salt-tolerant and normal lines of four grass species to $\mathrm{NaCl}$ in sand culture. New Phytology 104:453-461.

Ashraf, M., T. Mcneilly y A.D. Bradshaw. 1986c. The potencial for evolution of salt $(\mathrm{ClNa})$ tolerance in sevent grass species, New Phytology 103: 299-304.

Ashraf, M., T. Mcneilly y A.D. Bradshaw. 1987. Selection and heritability of sodium chloride in four forage grasses. Crop Science 227: $232-$ 234.

Ayers, A.D. 1950. Seed germination as affected of Soil Moisture and salinity. Agronomy Journal 44: 82-84.

Ayers, A.D. y H.E. Hayward. 1948. A method for measuring the effects of soil salinity on seed germination with observations on several crop plants. Soil Science Society of America Proceedings 13:224-226.

Blum, A. 1988. Plant breeding for stress environments. CRC Press, Boca Ratón/FL.

Bradford, K.J. 1995. Water realations in seed germination. Pages 351-396. In: J. Kigel and G. Galili (eds.). Seed Development and Germination. Marcel Dekker Inc. NY, USA.

Cheeseman, J.M. 1988. Mechanisms of salinity tolerance in plants. Plant Physiology 87:547550.

Flowers, T.J. 2004. Improving crop salt tolerance. Journal of Experimental Botany 55:307-319.

Foolad, M.R. y R.A. Jones. 1991. Genetic analysis of salt tolerance during germination in Licopersicon. Theoretical Applied Genetics 81:321-326.

Foolad, M.R. y R.A. Jones. 1992. Parent-offspring regression estimates of heritability for salt tolerance during germination in tomato. Crop Science 32:439-442.

Greenway, H. 1965. Plant responses to soil substrates. VII. Growth and ion uptake throughout plant development in two varieties of Hordeum vulgare. Australian Journal Biological Science 18:763.

Guerrier, G. 1981. Influence de differentes salinités (sels de sodium et sels de chlorure) sur la germination de Raphanus sativus. Plant and Soil 61:457-469.

Hasegawa, P.M., R.A. Bressan, J.K. Zhu y H.J. Bohnert. 2000. Plant cellular and molecular responses to high salinity. Annual Review Plant Physiologiy Plant Molecular Biology 51:463-499.

Horst, G. L. y N.B. Dunning. 1989. Germination and seedling growth of perennial ryegrass in soluble salts. Journal of American Society Horticultural Science 114:338-342.

INTA-CIRN. 1989. Mapa de Suelos de la Provincia de Buenos Aires. Proyecto PNUD ARG 85/019. 
Buenos Aires , Argentina.

ISTA. 1999. International Rules for seed testing. Internacional Seed Testing Association (ISTA). Seed Science and Technology (Supplement) 27:1-333.

Lucero, J.C. 1970. Germinación de cuatro gramíneas forrajeras bajo distintas condiciones de salinidad. Revista de Información sobre Investigación y Desarrollo Agropecuario (INTA) 273:60-64.

Ma, S., Q. Gong y H.J. Bohnert. 2006. Dissecting sat stress pathways. Journal of Experimental Botany 57:1097-1107.

Maas, E.V. 1986. Salt tolerance of plants. Applied Agricultural Research 1:12-27.

Maas, E.V. y G.H. Hoffman. 1977. Crop salt tolerance current assessment. Journal of Irrigation and Drainage Division 103:115-134.

Maddaloni, J. 1986. Forage production on saline and alkaline soil in the humid region of Argentine. Reclamation and Revegetation Research 5:1116.

Marcar, N.E. 1987. SALT tolerance in the genus Lolium (ryegrass) during germination and growth. Australian Journal Agronomy Research 38: 297-307.

Miller, T.R. y S.R. Chapman. 1978. Germination responses of three forage grasses to different concentrations of six salts. Journal Range Management 31:123-124.

Munns, R. 2002. Comparative physiology of salt and water stress. Plant, Cell and Environment 25:239-250.

Munns, R. 2005. Genes and salt: bringing them together. New Phytology 167:645-663.

Munns, R., R.A. James y A. Lauchli. 2006. Approaches to increasing the salt tolerance of wheat and other cereals. Journal of Botany 57:1025-1043.

Norlyn, J.D. y E. Epstein. 1984. Variability in salt tolerance of four triticale lines at germination and emergence. Crop Science 24:1090-1092.

Pearen, J.R., M.D. Pahl, M.S. Wolynetz y R. Hermesh. 1997. Association of salt tolerance at seedling emergence with adult plant performance in slender wheatgrass. Canadian Journal of Plant Science 77:81-89.

Quinn, J. y J. Colosi. 1977. Separating genotype from environment in germination ecology studies. The American Midland Naturalist 97:484-489.

Rogers, M.E., C.M. Grieve y M. Shannon. 1998. The response of lucerne (Medicago sativa) to sodium sulphate and chloride salinity. Plant and Soil 202:271-280.

Roundy, B.A. 1983. Response of basin wildrye and tall wheatgrass seedlings to salination. Agronomy Journal 75:67-71.

Rumbaugh,M.D.y B.M.Pendery. 1990.Germination salt resistence of alfalfa (Medicago sativa) germplasm in relation to subspecies and centers of diversity. Plant Soil 124:47-51.

Saleki, R., P.G. Young y D.D. Lefebvre. 1993. Mutants of Arabidopsis thaliana capable of germination under saline conditions. Plant Physiology 101:839-845.

Shannon, M.C. 1979. In quest of rapid screening techniques for plant salt tolerance. Horticultural Science 14:587-589.

Shannon, M.C. 1985. Principles and strategies in breeding for higher salt tolerance. Plant and Soil 89:227-241.

Shannon, M.C. y C.L. Noble. 1995. Variation in salt tolerance and ion accumulation among subterranean clover cultivars. Crop Science 35:798-804.

SSSA. 1997. Glossary of Soil Science Terms. Soil Science Society of America (SSSA), Madison, WI., USA.

Yeo, A.R. 1998. Molecular biology of salt tolerance in the context of whole-plant physiology. Journal of Experimental Botany 49:915-929. 